\title{
Microbiological Profile and Antibiotic Sensitivity Pattern In Community Acquired Urinary Tract Infection: Study From A Tertiary Care Hospital
}

\author{
Pradip Kumar Behera, ${ }^{1}$ Krishna Padarabinda Tripathy ${ }^{2}$, Surya Narayan Mishra ${ }^{3}$, \\ Ranjita Panigrahi ${ }^{4}$ \\ ${ }^{l}$ Assoc. Prof. , Dept. Of Medicine, Kalinga Institute Of Medical Sciences, Bhubaneswar, India \\ ${ }^{2}$ Assoc. Prof. , Dept. Of Medicine, Kalinga Institute Of Medical Sciences, Bhubaneswar, India \\ ${ }^{3}$ Asst. Prof. , Dept. Of Microbiology, Kalinga Institute Of Medical Sciences, Bhubaneswar, India \\ ${ }^{4}$ Assoc. Prof. , Dept. Of Pathology, Kalinga Institute Of Medical Sciences,Bhubaneswar, India
}

\begin{abstract}
:
Background: Urinary tract infection (UTI) is one of the common infections encountered by the clinicians. Though a good number of antimicrobial agents are available, still UTIs have become difficult to treat due to development of resistance by the uropathogens. So regional data regarding the common uropathogens and their sensitivity pattern is required to guide the clinicians to start empiric therapy while managing UTIS.

Objectives: The aim and objective of this study was to detect the common organisms causing community acquired UTI in our region which is a part of Eastern India and their sensitivity and resistance pattern towards different commonly used antimicrobial agents.

Material and methods: The present study is a prospective observational study carried out in a tertiary care teaching hospital in Odisha, India for a period of one year. Only hospitalized patients were included in the study after exercising the inclusion and exclusion criteria. A total of 240 samples were tested for bacteriological and antibiotic sensitivity study using standard procedures.

Results: Out of 240 urine samples, 124 samples tested positive for culture .The most common isolate was E. coli accounting for $40 \%$ of the total isolates. Klebsiella pneumoniae(24\%), Enterococcus Species (16\%), Pseudomonas aeruginosa (6\%) and Staph. aureus (6\%) were the other common isolates. E. coli which was responsible for highest number of cases was found to be sensitive to colistin (94\%), Amikacin(84\%) ,Nitrofurantoin (80\%) and Imipenem (72\%) but was resistant to antimicrobials like Ampicillin(88\%), Amoxicillin(72\%), Cefuroxime(88\%) and Ciprofloxacin(88\%).

Conclusion: Our study showed that common organisms causing Community acquired UTI are resistant to antimicrobials frequently prescribed by clinicians like Ampicillin, Amoxicillin,Cefuroxime, Ciprofloxacin and Levofloxacin and should be avoided .Nitrofurantoin and Amikacin are still effective against a good number of uropathogens and can be considered for empiric therapy. Broad spectrum antibiotics like Colistin, Imipenem, Tigecycline, Teicoplanin, Pip-Taz and Linezolid have shown effectiveness but should be judiciously used in appropriate situation and at proper dosage to avoid development of resistance.
\end{abstract}

Keywords: Community acquired,Urinary tract infection, Antibiotic susceptibility, Antibiotic resistance

\section{Introduction}

Urinary tract infection( UTI) is one of the most common infections encountered worldwide exceeded in frequency only by respiratory and gastrointestinal infections. ${ }^{1}$ Worldwide, about 150 million people are diagnosed with UTI each year, costing the global economy in excess of 6 billion US dollars. ${ }^{2}$ Neonates, girls, young females and elderly male are most susceptible to UTI.As many as $50-80 \%$ of women in the general population acquire at least one UTI during their life time and about $20-30 \%$ of women who have had one episode of UTI will have recurrent episodes. ${ }^{3}$ With advancing age incidence of UTI increases in men due to prostate enlargement and neurogenic bladder. Cases of UTI are usually treated with antibiotics empirically before the laboratory results of urine culture and sensitivity patterns are available. But the spectrum of microbiologic agents causing urinary tract infection and their antimicrobial sensitivity pattern have been continuously changing over the years both in community and in hospitals. ${ }^{4}$ Various studies done in different parts of world, have documented changing patterns of microbiological etiology of urinary tract infections. ${ }^{5,6}$ Antibiotic resistance is a serious health problem particularly in the developing world. Frequent use of broad spectrum antibiotics over specific antibiotics, poor patient compliance and incomplete course of antibiotics has added to the rapid evolution of antimicrobial resistance. ${ }^{7}$ Therefore it is important to have hospital based knowledge of the organisms causing UTI and their sensitivity pattern which is essential to formulate guidelines for the empiric treatment of UTIs while awaiting the culture sensitivity. The aim of the present study was to 
know the microbiologic etiology of community acquired urinary tract infections in our region which represents Eastern part of India and to study the antibiotic sensitivity pattern which can guide rational antibiotic use for benefit of the patients as well as to avoid drug resistance.

\section{Material And Methods}

The study was carried out in the Dept Of Medicine, Kalinga Institute of Medical sciences(KIMS) ,Bhubaneswar, India over a period of 1 year during the period from April 2015 to March, 2016 after due approval of the Institutional ethics committee. A total of 240 patients admitted to different wards of Dept of Medicine with clinical diagnosis of UTI or asymptomatic patients with significant number of pus cells in urine or nitrite positivity were enrolled in the study. Patients with history of antibiotic use in preceding 15 days, Hospitalization in last 3 days or patients developing symptoms after $48 \mathrm{hr}$ of hospitalization or history of any kind of urosurgery in last 30 days were excluded from the study. Relevant Clinical history and other data were collected in the patient data form. Every enrolled patient was educated regarding collection of mid stream urine sample and was provided with a sterile container with screw-cap. Clean catch mid-stream urine samples were collected in the sterile containers, sent within 2 hours to the central laboratory. Urine samples were processed in the Microbiology section of Central Laboratory. A preliminary screening of the uncentrifuged urine was done by making a wet-mount and gram stain to observe the polymorphs and the probable pathogen. The samples were then plated as per standard guideline on CLED and Mac Conkey Agar plates and were incubated at $37^{\circ} \mathrm{C}$ overnight. The colony growth was observed and the CFU/Ml was noted and are processed to identify the organism both manually and using automated method (BactT alert and Vitek2). The manual sensitivity was also put using the Kirby Bauer's multiple disc diffusion method following standard precaution .Antibiotics commonly used in clinical practice for treatment of urinary tract infection were chosen for sensitivity test. Care was taken to include antibiotics which will cover both gram positive as well as Gram negative organisms. Broad spectrum antibiotics frequently used in empiric therapy of severe infections in ICU set ups were given special importance. After getting all the reports statistical analysis was done.

\section{Results}

Out of 240 urine samples sent for culture and sensitivity test 124 samples showed significant growth of single micro-organisms with a culture positivity rate of $51.66 \%$. Those with growth of more than one microorganisms were considered as contaminants and discarded. Patients were in the age range of 17 year to 85 year. Table -1 shows the age and sex distribution of the study sample.Table $-1(n=124)$

\begin{tabular}{|l|l|l|l|}
\hline Age in yrs & Male & Female & Total \\
\hline$<20$ & 0 & 3 & 3 \\
\hline $20-39$ & 5 & 23 & 28 \\
\hline $40-59$ & 12 & 20 & 32 \\
\hline $60-79$ & 30 & 18 & 48 \\
\hline$>80$ & 8 & 5 & 13 \\
\hline Total & 55 & 69 & 124 \\
\hline
\end{tabular}

Majority of patients were in the age range of 60 to 79years (39\%) followed by 40 to 59 years (32\%). In the study group over all females outnumbered males at a ratio of 1.2:1.But in the elderly age group ( $>60$ yrs) male patients represented more than females. $(M=62 \%$,Female $=38 \%)$.

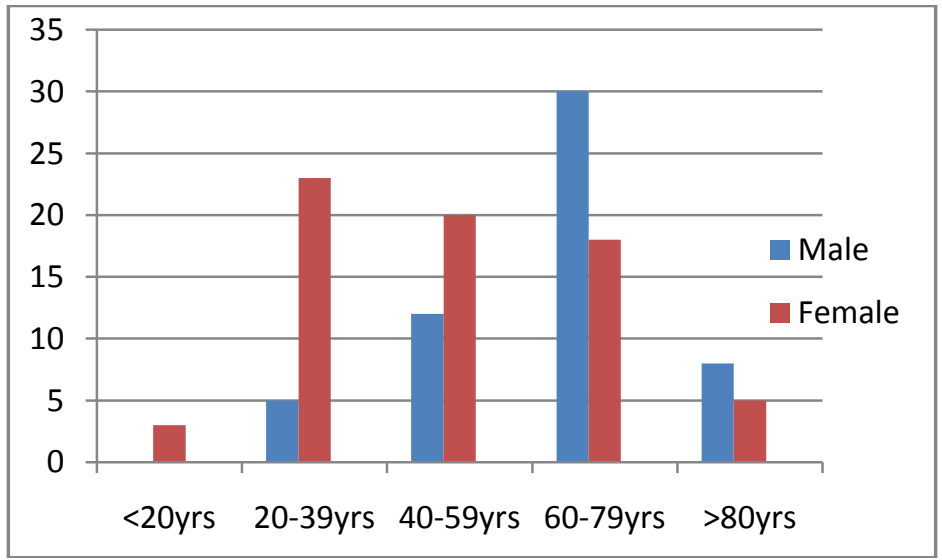

Fig. 1 Age and sex distribution of study population 
$58 \%$ patients in the study population were diabetic and $52 \%$ were having hypertension. Among elderly males $62 \%$ patients were having symptomatic BEP. The organisms isolated, were mostly Gram negative aerobic rods (>70\%).Five most common isolates were E.coli (40\%), Klebsiella pneumoniae (24\%), Enterococcus Species (16\%), Pseudomonas aeruginosa(6\%) and Stapylococcus aureus(6\%)

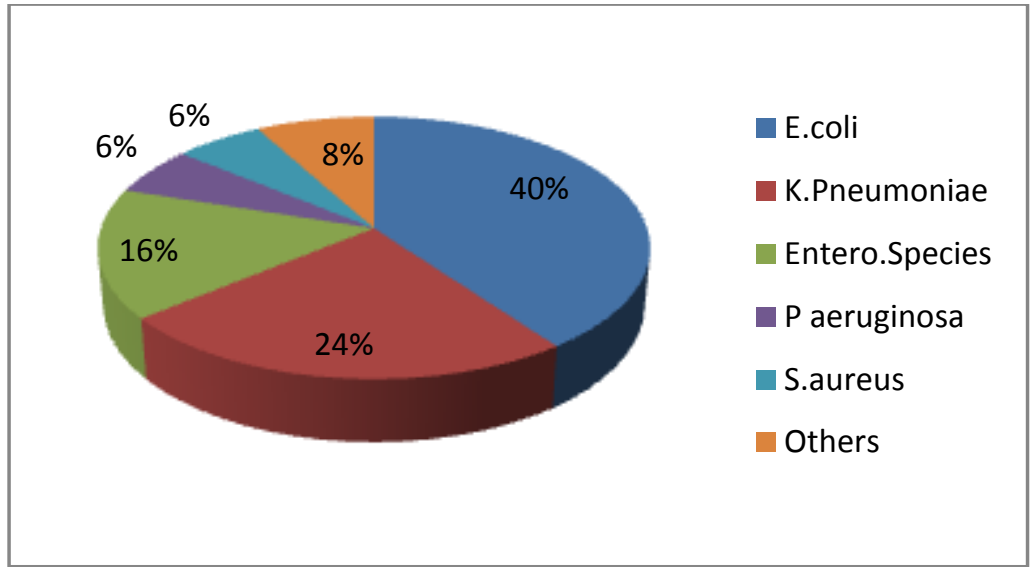

Fig: 2 showing distribution of common isolates.

The most common isolate, E. Coli showed $94 \%$ sensitivity to colistin.Other antibiotics to which $E$. coli was sensitive were Amikacin 84\%, Imipenem-72\%, Nitrofurantoin-80\% and Piperacillin -Tazobactam$56 \%$ The antibiotics to whom E. coli was resistant were Ampicillin-88\%, Cefuroxime-88\%, Ciprofloxacin 88\%, ceftriaxone $76 \%$ and Amoxicilin-72\%.

Table -2. Showing antibiotic sensitivity and resistance pattern of five most common isolates

\begin{tabular}{|l|l|l|}
\hline $\begin{array}{l}\text { Organisms } \\
\text { isolated }\end{array}$ & Antibiotics sensitivty & Antibiotic resistance \\
\hline E. coli & Colistin-94\% & Ampicillin-88\% \\
& Amikacin -84 $\%$ & Cefuroxime-88\% \\
& Nitrofurantoin-80\% & Ciprofloxacin-88\% \\
& Pmipenem-72\% & Ceftriaxone-76\% \\
& Aip-Taz-56\% & Amoxicillin-72\% \\
\hline K. pneumonie & Tigecycline-86\% & Ampicillin-100\% \\
& Colistin-80\% & Cefuroxime-93\% \\
& Imipenem-60\% & Ceftriaxone-80\% \\
& Amikacin-53\% & Amoxicillin- 80\% \\
& Nalidixic acid-53\% & Nitrofurantoin-53\% \\
\hline Entero. fecalis & Teicoplanin-96\% & Ciprofloxacin-100\% \\
& Tigecycline-92\% & Levofloxacin-86\% \\
& Vancomycine-90\% & Tetracycline-84\% \\
& Linezolid-90\% & Erythromycin-90\% \\
& Nitrofurantoin-70\% & Benzylpenicillin-40\% \\
\hline P .aerogenosa & Imipenem-90\% & Ampicilin-100\% \\
& Colistin-90\% & Amoxicillin-84\% \\
& Pip-Taz-70\% & Cefuroxime-76\% \\
& Amikacin-60\% & Tigecycline-60\% \\
& Ciprofloxacin-60\% & Nitrofurantoin-60\% \\
\hline Staph aureus & Linezolid-96\% & Ciprofloxacin-100\% \\
& Teicoplanin-94\% & Levofloxacin-72\% \\
& Vancomycin-94\% & Ampicillin-100\% \\
& Nitrofurantoin-90\% & Benzylpenicillin-82\% \\
& Tetracycline-80\% & Amoxicillin-80\% \\
\hline
\end{tabular}

Klebsiella Pneumoniae was the second most common isolate and was most sensitive to Tigecycline( $86 \%)$.Other antibiotics to which Klebsiella Pneumoniae was sensitive were colistin (80\%), Imipenem 60\%), Amikacin and Nalidixic acid( both $53 \%$ sensitive).Antibiotics to which K. pneumoniae was resistant were in order of frequencyAmpicilin,(100\%), cefuroxime(93\%),Ceftriaxone(80\%),Amoxicilin(80\%)and 
itrofurantoin(53\%).Enterococcous species was the third most common organism isolated in the study. Teicoplanin (96\%)and Tigecycline (92\%)were most effective against Enterococcous in our study followed by Vancomycin and Linezolid( $90 \%$ sensitivity for both of the antibiotics).Enterococcous species were highly resistant to Fluroquinolones like Ciprofloxacin (100\%)and Levofloxacin(86\%) and tetracycline(84\%) .Erythromycine resistance for Enterococcous species was observed in $90 \%$ cases. Pseudomonas aeruginosa was the next common organism isolated and Colistin (90\%), Imipenem(90\%),Meropenem(90\%), PiperacillinTazobactam (70\%) and Amikacin (60\%) were the antibiotics most commonly effective against it. Pseudomonas. aeruginosa was sensitive to Ciprofloxacin in $60 \%$ of cases and $100 \%$ resistant to Ampicilin, Amoxicilin-Clavulanic acid(84\%), Cefuroxime $(76 \%)$ and nitrofurantoin(60\%). Cefoperazone- sulbactam was acive against Pseudomonas. aerugenosa in $50 \%$ cases. Staphylococcous aureus was the $5^{\text {th }}$ most common organism isolted in our study and was most responsive to Linezolid(96\%), Vancomycin (94\%) and Teicoplanin(94\%).Nitrofurantoin(90\%) and Tetracycine( $80 \%)$ were also fairly active against Staph. Aureus. But Benzyl penicillin(82\%),Ampicillin(100\%), Ciprofloxacin(100\%), Levofloxacin (72\%) were ineffective against Staph. aureus.

\section{Discussion}

While treating urinary tract infection(UTI), antibiotic therapy is warranted for any symptomatic UTI. ${ }^{3}$ The choice of antimicrobial agent, its dose and duration of therapy depends on the site of infection, presence or absence of complicating conditions and local prevalence of microorganisms, and their antibiotic sensitivity pattern. Antimicrobial resistance varies from region to region and impacts the empiric therapy of urinary tract infection. Nitrofurantoin, Trimethoprim-Sulfamethoxazole, B-Lactam Antibiotics like Ampicillin, Amoxycillin, Cefuroxime, Ceftriaxone, Cefoperazone, Combinations of $\beta$-lactams with $\beta$-lactamase inhibitors like Amoxicillin-clavulanic acid or Cefoperazone-Sulbactam, fluroquinolones like Ciprofloxacin, ofloxacin, levofloxacin, aminoglycosides like Amikacin, Gentamycin are the antimicrobial agents commonly used by the clinicians while managing UTI of mild to moderate severity. In critical care setting while managing cases of UTI with urosepsis with or without multiorgan dysfunction intensivist largly depend on antimicrobial agents like Piperacillin- Tazobactam,Imipenem, Imipenem-cilastatin, Tigecycline, Colistin, Teicoplanin, Vancomycin or Linezolid. The empiric treatment of UTI will be more effective if the local prevalence of microorganisms and their sensitivity pattern is known.

In studies conducted by different researchers the culture positivity varies from $10.86 \%$ to $84 \%{ }^{8-12}$ and in our study the culture positivity was $51.66 \%$ which is similar to many other studies. Table-3 shows the culture positivity and three most common isolates observed different studies

Table-3 Showing culture positivity and four most common isolates in different studies

\begin{tabular}{|l|l|l|l|}
\hline SL no & Authors & $\begin{array}{l}\text { Culture } \\
\text { positivity }\end{array}$ & Organisms isolated in \% \\
\hline 1 & Shalini et al & $84.12 \%$ & E. coli-64.3 \\
& & & K. pneumoniae-20.3 \\
& & & P. aeruginosa-9.1 \\
S. aureus-6.3
\end{tabular}

The age group analysis showed that majority of our subjects were in the age group 60 to $79 y$ rs (39\%) followed by $40-59$ yrs $(32 \%)$. But in many other studies the common age group of the study population was young and middle age ( $<40 \mathrm{yrs})$.In their study M Dash et all observed 55.4\% subjects were between 18-37 yrs. ${ }^{13}$ Such difference in the age group of the subjects may be due to the fact that our study population were 
hospitalized patients where as in other studies the subjects were from the out-patient departments. Elderly patients usually have complicated UTI with need for hospitalization than younger patients who usually suffer from uncomplicated UTI. Female to male ratio in our study was 1.2:1 which was comparatively higher in other studies( Rijal et $\mathrm{al}^{11}-3.2: 1$, Dash et $\mathrm{al}^{13}-3.7: 1$ ). This difference of female dominance may be due to the fact that significant proportion of our patients were elderly and male subjects were more than female subjects in this age group.

In our study organisms isolated in decreasing order were E. coli-40\%,Klebsiella pneumoniae-24\%, Enterococcous species-16\%,Pseudomonas aerugenosa-6\%,Staph. aureus 6\%. Some of the less common isolates were Proteus mirabillis, Morganella morgani, Acenitobacter boumani and Candida albicans. The pattern of isolates are almost similar to observations in other studies as shown in the Table-6. In all the studies E. coli was the most common organism causing UTI and accounts for $31.25 \%$ to $64.33 \%$ of all isolates in various studies. In five out of six studies including our study, Klebsiella pneumoniae is the second most common organism causing UTI. In our study K. pneumoniae is responsible for $20 \%$ of total UTI cases and simillar results observed by Akram et al ${ }^{10}(22 \%)$ and Shalini et $\mathrm{al}^{8}(20.3 \%)$.Enterococcous speciesis the third most common isolate on our study which is similar to the observation of R N Das et al ${ }^{12}$ But Enterococous isolate was not so common in other studies. Pseudomonas and Staph aureus were the next common isolates in our study accounting for 6\% each. Nearly similar observations noted in other studies. Shalini et al ${ }^{8}$ observed Pseudomonas and Staph aureus isolates $9.1 \%$ and $6.3 \%$ respectively in their study. Akram et $\mathrm{al}^{10}$ in their study observed the presence of Pseudomonas and Staph aureus to be $7 \%$ and $4 \%$ rspectively. Only in one study (Durgesh et al ${ }^{9}$ ) Staph aureus and Pseudomonas isolates were higher(25\% and $15.62 \%$ respectively).

The E. coli isolates in our study showed highest sensitivity against Colistin(100\%) followqed by Amikacin(84\%) and Nitrofurantoin( 80\%).Imipenem and Piperacillin -Tazobactam also were effective against E.coli with $72 \%$ and $56 \%$ sensitivity respectively.This is similar to the results observed by shalini et $\mathrm{al}^{8}$ and Rijal et al $^{11}$ where E. coli isolates were sensitive to Amikacin $(98.91 \%)$ and Nitrofurantoin $(93.48 \%)$ .Commonly used antimicrobials like Ampicillin, Amoxicillin, Ciprofloxacin, Cefuroxime and Ceftriaxone were highly ineffective against E. coli with $>70 \%$ resistance. High resistance of E.coli against Amoxicilin and Ceftriaxone was also observed by Durgesh et $\mathrm{al}^{9}$, Shalini etal ${ }^{8}$, Akram et al ${ }^{10}$ and Rijal et $\mathrm{al}^{11}$ in their study.In contrary Nitrofurantoin resistance of E.coli which was observed by Akram et al ${ }^{10}$ from Aligarh , India in 2006 has changed in our study in 2015 where we observed high sensitivity of E.coli against Nitrofurantoin.This may be due to less use of Nitrofurantoin during this period has revived the sensitivity. High sensitivity of E.coli for fluroquinolones as observed by Shalini et $\mathrm{al}^{8}$ has now changed as evident in our study and other studies by Durgesh et $\mathrm{al}^{9}$ and Rijal et $\mathrm{al}^{11}$. This may be due to frequent use of these drugs for UTI in Indian sub-continent.

Klebsiella Pneumoniae, the second most common organism isolated in our study, showed highest sensitivity towards Tigecycline(86\%), Colistin (80\%) and Imipenem(60\%).Nalidixic acid and Amikacin were also effective. In their study Akram et $\mathrm{al}^{10}$ also observed high senitivity of Klebsiella for Imipenem and Amikacin. Klebsiella Pneumoniae resistnace for Nitrofurantoin and Tetracycline was observed in both the studies. In our study high resistance of Klebsiella pneumoniae noted against $\beta$-lactams like Ampicillin, Amoxycillin, ceftriaxone and cefuroxime which is similar to observations of Shalini et al ${ }^{8}$. But Fluroquinolones which were highly active against Klebsiella pneumoniae as observed by Shalini et al ${ }^{8}$ were found to be less effective in our study. Studies from different parts of the world shows that E.coli and Klebsiella species are still the commonest uropathogens isolated in community aquired UTI. ${ }^{15-18}$

Enterococci are well-known as nosocomial opportunistic pathogens but Enterococcus isolates were $16 \%$ of all isolates in our study where as it was less common isolate in other studies ( Akram et all 1\%, Das RN et al $8.1 \%$ ).In our study Enterococcal isolates were having high sensitivity towards Teicoplanin(100\%), Tigecycline(100\%), Linezolid(90\%), Vancomycin(90\%) and Nitrofurantoin( $70 \%)$.In their study, Goel et al ${ }^{19}$ observed E. feacalis to have highest sensitivity for Linezolid(100\%) followed by Teicoplanin (88.5\%), Nitrofurantoin(86\%) and Vancomycin(77.1\%). In both the studies E .feacalis was resistant to Tetracyline and Quinolones. Pseudomonas isolates in our study were highly susceptible to antibiotics like Colistin(90\%), Imipenem(90\%), Pip-Tazobactam(70\%), Amikacin(60\%) and ciprofloxacin(60\%). Similar observations noted in study by Shalini et al ${ }^{8}$ (Amikacin-61.5\%,Norfloxacin-76.92\%, ciprofloxacin-69.32\%) . Study by Alka Nerurkar et $\mathrm{al}^{14}$ observed high resistance of Pseudomonas against Ampicillin,Co-Trimoxazole and Norfloxacin. Our study also revealed similar findings with Ampicillin, Amoxicillin and cefuroxime being very much ineffective towards Pseudomonas. Staphylococcous isolates in our study showed high susceptibility for Linezolid (100\%), Teicoplanin (100\%) Vancomycin (96\%), Nitrofurantoin (90\%), Tetracycline (86\%) but were resistant against Ciproflcxacin, Levofloxacin and Benzyl penicillin.

Our study has revealed that commonly used oral antibiotics for community aquired UTI like Ampicillin, Amoxicillin, Ciprofloxacin, Levofloxacin and Cefuroxime have lost their effectiveness against most frequent uropathogens .Development of resistance by the uropathogens may be due to frequent unnecessary use of antibiotics in inappropriate dosage in our region. Only one oral antibiotic to which maximum 
number of organisms were sensitive was Nitrofurantoin. Among broad spectrum antibiotics Colistin , Tigecycline, Imipenem and Amikacin were more effective against Gram negative pathogens and Teicoplanin, Linezolid and Vancomycin showed high effectiveness against Gram positive organisms. So any of the above drugs can be chosen for empiric use in critical care settings.

\section{Conclusion}

Most of the studies including our study has revealed that the organism commonly causing community acquired urinary tract infection is E. coli. So the empiric antibiotic therapy should be chosen amongst one which are most effective against E coli. As Nitrofurantoin and Amikacin showed good activity against most organisms, clinicians may consider these drugs in uncomplicated Community acquired urinary tract infections. Imipenem, Colistin and Tigecycline may be considered in severe urosepsis in ICU set ups where choosing the right antimicrobial is life saving.Teicoplanin and Vancomycin should be considered where Staph. aureus is suspected. But antibiotic resistance among bacteria is a continuous and evolving process, regular surveillance and monitoring is required. The updated knowledge will help the physicians to use appropriate antibiotics for greater benefit of the patients. To prevent or decrease resistance physicians also should use the appropriate antibiotics at right doses for an appropriate period of time.

\section{References}

[1]. Levi ME, Redington J, Barth L. The patient with urinary tract infection. Manual of Nephrology. $6^{\text {th }}$ Edition. Lippincott Williams \& Wilkins.2005;7:91

[2]. Rock W, Schaeffer AJ. Treatment of Urinary tract infections: Whats old, Whats new and What works. World J Urol. 1999;17:37282.

[3]. K Gupta, BW Trautner. Urinary Tract Infections, Pyelonephritis and Prostatitis.In: Denis L Kasper,Anthony S Fauci,Stephen L Hauser, Dan L Longo, J L Jameson, J Loscalzo editors. Harrison's Principles of Internal Medicine. Vol. 2, 19 ${ }^{\text {th }}$ Ed $^{\text {n }} .861-$ 868.McGraw Hill Education.

[4]. Kahlmeter G. ECO.SENS. An Internationalsurvey of the antimicrobial susceptibility of pathogens from uncomplicated urinary tract infections: The ECO.SENS Project. J Antimicrobial Chemothr. 2003;51:69-76

[5]. Manges AR, Natarajan P, Solberg OD, SietrichPS, Riley LW. The changing prevalence of drug-resistant Escherchia coli clonal groups in acommunity:evidence for community out breaks of urinary tract infections. Epidemiol Infect. 2006;134: 425-31.

[6]. Kahan NR,Chintiz DP, Waitman DA,Dushnitzky D, Kahan E, Shapiro M. Empiric treatment of uncomplicated urinary tract infectionwith fluroquinolones in older women in Israel: another lost treatment option? Ann Pharmacother. 2006;40:2223-7.

[7]. Goldstein FW. Antibiotic susceptibility of bacterial strains isolated from patients with community acquired urinary tract infections in France. Multicenter study group. Eur J Clin Microbiol Infect Dis.2000;19:112-7

[8]. Shalini, Joshi MC, Rashid MK, Joshi HS. Study of Antibiotic Sensitivity pattern In Urinary Tract Infection At A Tertiary Hospital. NJIRM 2011;Vol.2(3).July-Sept. 43-46.

[9]. Durgesh D. Washnik, P M Tumane. Prevalence and antibacterial susceptibility pattern of Urinary Tract Infection causing Human Pathogenic Bacteria. Asian Journal of Biomedical and Pharmaceutical Sciences. 2(15);2012:1-3

[10]. Akram M, Shahid M, Khan AU.Etiolog and antibiotic resistance patterns of community -aquired urinary tract infections in JNMC Hospital, Aligarh, India. Ann Clin Microbiol Antimicrob. 2007;6:4

[11]. Rijal A, Ghimire G, Gautam K, Barakoti K. Antibiotic susceptibility of organisms causing urinary tract infection in patients presenting to a teaching hospital. J Nepal Health Res Counc 2012 Jan;10(20):24-7

[12]. Das RN, Chandrasekhar TS, Joshi HS, Gurung M, Shrestha N, Shivananda PG. Frequency and susceptibility profile of uropathogens causing urinary tract infections at a tertiary care hospital in Western Nepal. Singapore Med J. 2006 Apr;47(4):281-5

[13]. Muktikesh Dash, Sanghamitra Padhi,Indira Mohanty, Pritilata Panda and Banojini Parida. Antimicrobial resistance in pathogens causing urinary tract infections in a rural community of Odisha, India. Family community Med 2013.Jan-Apr;20(1):20-26.

[14]. Dr Alka Nerurkar, Dr Priti Solanky, Dr Shanta S. Naik. Bacterial pathogens in Urinary tract infection and Susceptibility pattern. Journal of Pharmaceutical and Biomedical Sciences.2012;21(12)

[15]. Kothari A, Sagar V. Antibiotic resistance in pathogens causing community-acquired urinary tract infections in India: A multicentre study. J Infect Developing Countries.2008;2:354-8.

[16]. Selvakumar BN, Jasmine R. Antibiotic susceptibility of ESBL-producing urinary isolates at a tertiary care hospital in Tiruchirapalli, South India.J Med Sci.2007;7:443-6.

[17]. Bahhadin J,Teo SS, Mathew S. Aetiology of community acquired urinary tract infection and antimicrobial susceptibility patterns of uropathogens isolated. Singapore Med J.2011;52:415-20

[18]. Bano K,Khan J,Begum RH,Munir S, Akbar N,Ansari J et al.Patterns of antibiotic sensitivity of bacterial pathogens among urinary tract infections (UTI) patients in Pakistani Population. Afr J Microbiol Res.2012;6:414-20. 